\title{
Lupus nephritis with an unusual histopathological pattern
}

\author{
Marcelo Aveiro, Nicole Pestana, Francisca Silva, Pedro Vieira, Nuno Rosa, Gil Silva \\ Nephrology Department, Hospital Central do Funchal, Funchal, Portugal
}

\section{ABSTRACT}

Introduction: Systemic lupus erythematosus (SLE) is an autoimmune disease that can affect multiple organs. When the kidney is involved it is generally termed lupus nephritis (LN), and is a major contributor to the morbidity and mortality associated with SLE. The classic pattern of lupus nephritis in a renal biopsy is an immune complex mediated glomerulonephritis. The presence of crescents, in diffuse proliferative lupus nephritis, and serologic positivity for antineutrophil cytoplasmatic antibodies (ANCA), more often antimyeloperoxidase than anti-proteinase 3 , has been linked with specific clinicopathological features, poor treatment response and a worse kidney survival.

Clinical case: We present the case of a 19-year-old male, without relevant past medical history, who presented severe headaches, hypertension and peripheral edema. The blood analysis revealed hemoglobin $(\mathrm{Hb}) 7.6 \mathrm{~g} / \mathrm{dL}$, creatinine $(\mathrm{Cr}) 2.64 \mathrm{mg} / \mathrm{dL}$, blood urea nitrogen (BUN) $101 \mathrm{mg} / \mathrm{dL}$, and urine analysis, hematuria and nephrotic proteinuria. The autoimmunity panel results were consistent with SLE paired with positivity for ANCA-proteinase 3 antibody. A renal biopsy revealed crescentic glomerulonephritis with fibrinoid necrosis and Bowman capsule rupture. The patient was diagnosed with class IV LN. The initiation treatment consisted of cyclophosphamide (CIPH) and prednisolone (PDN). At the 6-month follow-up, CIPH was stopped and mycophenolate mofetil (MMF) initiated as maintenance therapy, combined with PDN. Although microscopic hematuria and C3 consumption were still present (a new biopsy was pondered but the patient refused any further invasive diagnostic measures), $\mathrm{C} 1 \mathrm{q}$ levels decreased from 26.6 to $19.3 \mathrm{U} / \mathrm{ml}$ (negative if $<20 \mathrm{U} / \mathrm{ml}$ ) and anti-dsDNA was also negative with progressive declination of the degree of proteinuria. The creatinine levels returned to normal.

Conclusion: The authors emphasize the importance of this class IV LN, influenced by the association with anti-proteinase 3, due to the implications in the histopathological pattern and in therapy selection. In this specific case kidney function, proteinuria and lupus activity had an important decrease without significant complications with the chosen treatment.

Keywords: Lupus Nephritis, Cyclophosphamide, Crescentic Glomerulonephritis

\section{INTRODUCTION}

Systemic lupus erythematosus (SLE) is a chronic autoimmune disease that can cause inflammation and damage to any organ system. It is more prevalent in women than men across all age groups. Lupus nephritis (LN) is a major risk factor for morbidity and mortality in SLE and occurs in about $20-65 \%$ of patients, which may lead to end-stage renal disease (ESRD) in about $10 \%$ of those affected ${ }^{1}$. Male patients tend to have more frequent renal involvement and greater severity of disease 2,3 . The classic pattern of lupus nephritis in a kidney biopsy is an immune complex mediated glomerulonephritis. It has been found that ANCA positivity, more often antimyeloperoxidase (82\%) than anti-proteinase $3(7 \%)$, influences the histological pattern of lupus nephritis and is associated with worse baseline renal function and more active lupus serology 4 . The pathogenesis of ANCA in LN patients is still not clear. It was observed that, when ANCA is present, other autoantibodies appeared more frequently. This could be explained due to neutrophil extracellular trap (NET) formation (released by NETosis) ${ }^{5}$. These NETs could serve as a font of autoantigens that leads to the production of pathogenic antibodies, contributing to the progression of LN. Also, ANCA could play a role in the activation of the complement pathway in contributing to the pathogenesis of $\mathrm{LN}^{5}$. The presence of crescents, in diffuse proliferative lupus nephritis, has been linked with specific clinicopathological features, a poor treatment response and a worse kidney survival ${ }^{6}$. Therefore, the choice of treatment is crucial when trying to achieve complete remission, which is defined as urinary protein excretion $<0.3 \mathrm{~g} /$ day, normal urinary sediment, and normal or stable renal function (within $10 \%$ of normal eGFR if previously abnormal renal function), in these severe cases ${ }^{7}$.

\section{CLINICAL CASE DESCRIPTION}

The authors present the case of a 19-year-old Caucasian male, without any relevant past medical history, who presented to the emergency department complaining of severe headaches for 5 days. The physical examination revealed peripheral edema and grade 2 hypertension (167/72 $\mathrm{mmHg}$ ). The blood analysis revealed a normocytic normochromic anemia (Hg 7.6 g/dL; MCV 75 fL; MCHC 35g/dL), with $\mathrm{Cr}$ of $2.64 \mathrm{mg} / \mathrm{dL}$ and BUN of $101 \mathrm{mg} / \mathrm{dL}$, an acute kidney injury Stage II KDIGO: Kidney Disease: Improving Global Outcomes, and creatinine kinase of $1024 \mathrm{U} / \mathrm{L}$. Hematuria (++) and proteinuria $(100 \mathrm{mg} / \mathrm{dL})$ were also present in type II urinalysis. The cranial computerized tomography was normal. The patient was admitted to a general ward for treatment and etiological investigation. The investigation was remarkable for antibodies positivity for antinuclear (ANA), ANCA-proteinase 3, antidouble stranded DNA (Anti-dsDNA), anti-histone, anti-Smith, proliferating cell nuclear antigen (anti-PCNA) as well as low C3, C4 and 
Table 1

Principal variables response to the chosen therapeutic strategy at follow-up

\begin{tabular}{l|c|c|c|c|c}
\multicolumn{1}{c|}{ Variable } & Normal range & Admission & 6-month & 12-month & 18-month \\
\hline Creatinine (mg/dL) & $0.7-1.2$ & 2.64 & 1.6 & 1.4 & 1.1 \\
\hline Total protein (mg/24h) & $<0.15 g$ & 6800 & 600 & 250 & 100 \\
Ac. Anti-dsDNA IgG (IU/mL) & $>50$ & 304 & 25 & 20 & 26 \\
Ac. Anti-C1q (U/ml) & $<20$ & 26.6 & 19.7 & 8.3 & \\
C3 (mg/dL) & $90-180$ & 68 & 78 & 80 & \\
C4 (mg/dL) & $10-40$ & 3,5 & & \\
CH50 & $42-95$ & $32 \%$ & & 4 & \\
Ac. Anti-Proteinase 3 (U/mL) & $<8$ & 23 & 6 & & \\
& & & &
\end{tabular}

CH50 levels (Table 1 ). The 24-hour urine analysis revealed proteinuria in the nephrotic range $(6.9 \mathrm{~g} / 24 \mathrm{~h})$. A renal biopsy (Fig. 1-4) was then performed. There were 15 glomeruli in the fragment and 10 of them presented medium to large crescents, ranging from cellular to fibrotic, adherent to Bowman's capsule. Eight of these glomeruli presented with rupture of Bowman's capsule and 6 presented fibrinoid necrosis and presence of karyorrhexis. Immunofluorescence was positive for IgG and C3 (Fig. 4). These deposits were of granular type along the glomerular wall in a segmental way. C1q deposits were not evident. Lack of glomeruli in the frozen segment meant immunofluorescence was performed in the paraffin fragment. This technique is less sensitive and therefore false negatives may exist. Electron microscopy was not performed. The patient was diagnosed with class IV Lupus Nephritis with modified NIH (National Institute of Health) Activity Index Score of 22 and Modified NIH Chronicity Index of 0 . The initiation treatment of choice consisted of intravenous cyclophosphamide $1.0 \mathrm{~g} / \mathrm{m} 2(\mathrm{CIPH})$, monthly during 6 consecutive months, in association with prednisolone $(1 \mathrm{mg} / \mathrm{kg} /$ day) with slow tapering. The patient was discharged and

\section{Figure 1}

Methenamine silver 200X. Fibrinoid necrosis and cellular crescents.

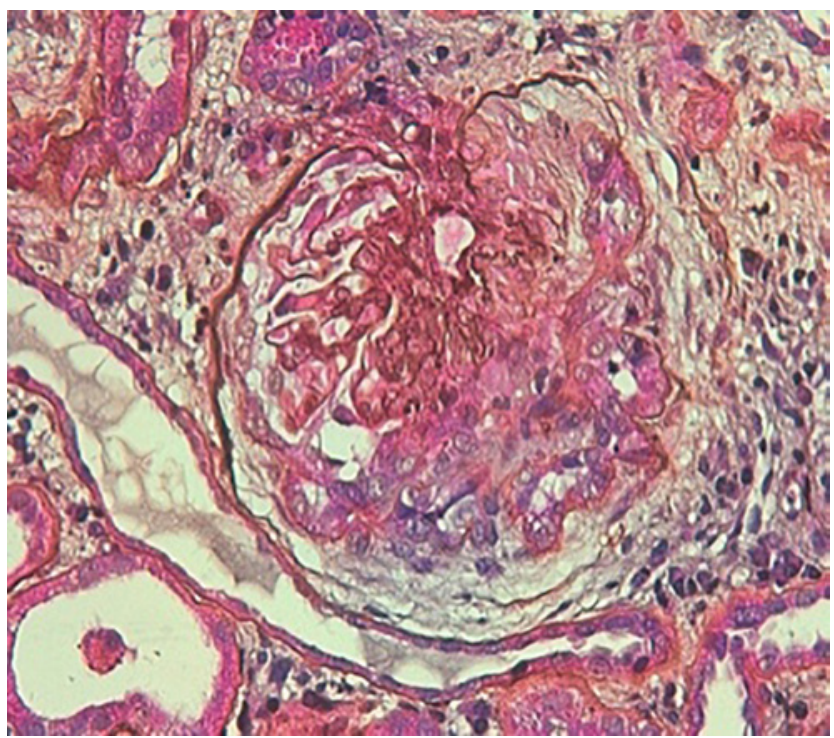

\section{Figure 2}

Masson trichrome 200X. Circumferential fibrocellular crescents. Bowman's capsular rupture.

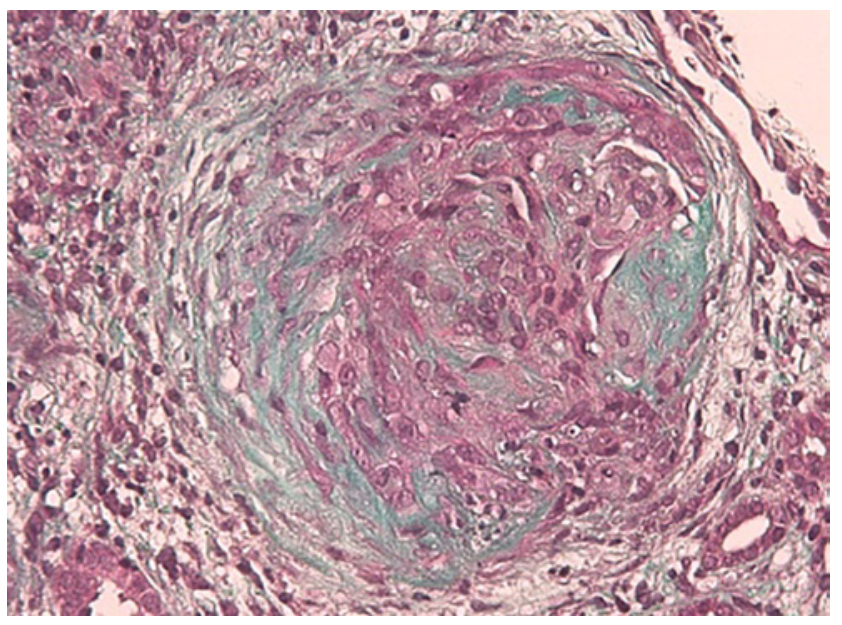

\section{Figure 3}

Periodic acid Schiff 200X. Cellular crescents.

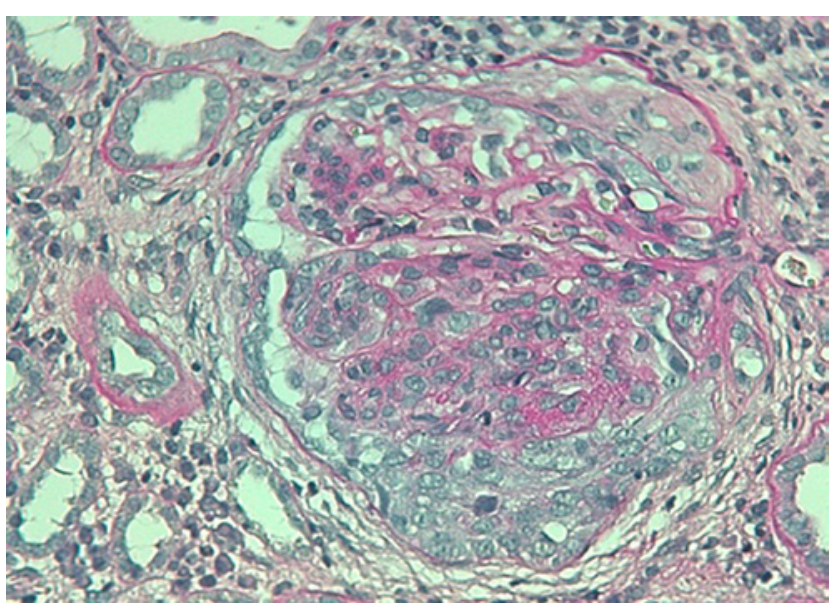




\section{Figure 4}

Immunofluorescence revealing granular, segmental deposits along the glomerular wall.

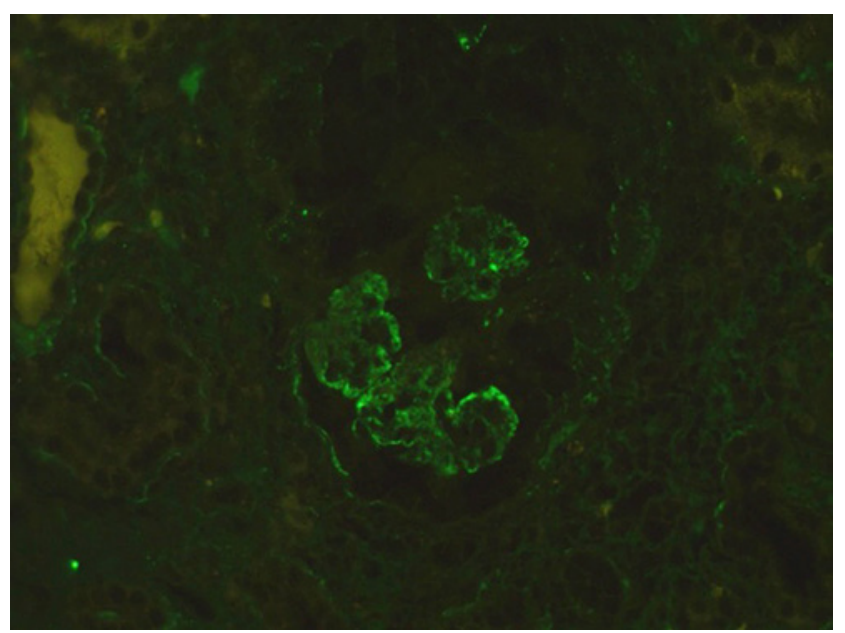

was closely monitored via regular medical appointments. At 6-month follow-up CIPH was stopped and mycophenolate mofetil (MMF) (1g twice daily) initiated, as maintenance therapy, combined with prednisolone. Although there were some signs of disease activity, such as microscopic hematuria, and C 3 consumption were still present (a new biopsy was pondered but the patient refused any further invasive diagnostic measures), C1q levels decreased from 26.6 to $19.3 \mathrm{U} / \mathrm{ml}$ (negative if $<20 \mathrm{U} / \mathrm{ml}$ ). ANCA-proteinase 3 and anti-dsDNA were negative. The degree of proteinuria progressively declined and reached $<300 \mathrm{mg} /$ day eight months after treatment onset. The creatinine values had a slow descent, reaching normal levels $(1.2 \mathrm{mg} / \mathrm{dL})$ after eighteen months of treatment (shown in Table 1). During this period the patient maintained MMF and prednisolone was gradually reduced to $5 \mathrm{mg}$ and then suspended.

\section{DISCUSSION}

Cyclophosphamide $(0.5-1 \mathrm{~g} / \mathrm{m} 2$, monthly dose for 6 months, (also known as National Institute of Health (NIH) protocol) was the first immunosuppressive treatment that proved superior to a single-corticosteroids strategy ${ }^{8-11}$ when treating LN. A lower-dose strategy (500 mg iv every 2 weeks for 3 months), also known as Euro-Lupus regimen, demonstrated a similar efficacy to the previously stated regimen ${ }^{12,13}$; however, there was a low number of patients in the trial with widespread ( $>50 \%$ ) segmental glomerular necrosis or crescents ${ }^{14}$. In some cases, MMF has been found to have an equal response rate to $\mathrm{CIPH}$ at 6 months, with no major differences in adverse effects ${ }^{15}$. The Kidney Disease: Improving Global Outcomes (KDIGO), the American College of Rheumatology Guidelines for Screening, Case Definition, Treatment and Management of Lupus Nephritis and the Joint European League Against Rheumatism and European Renal Association-European Dialysis and Transplant Association (EULAR/ERA-EDTA) guidelines all recommend for the treatment of Class III and IV or proliferative lupus nephritis an initiation therapy which include glucocorticoids associated with either $\mathrm{CIPH}$ or $\mathrm{MMF}^{14,16,17}$. The chosen strategy will depend on several factors such as race, disease activity and personal preference. MMF may be preferred in young men and women due to high risk of testicular and ovarian failure following treatment with cyclophosphamide ${ }^{18}$. A study has found that patients with antineutrophil cytoplasmic antibody positivity serology were more likely to receive cyclophosphamide as treatment for their more active $\mathrm{LN}^{4}$. Also, in patients that present crescents on renal biopsy, an indication of severe LN, cyclophosphamide may be preferred, as it is one of the few drugs that has proven to be effective ${ }^{19,20}$. For maintenance therapy, the KDIGO guideline recommendations state that patients with class III and IV LN receive maintenance therapy with azathioprine (1.5-2.5 $\mathrm{mg} / \mathrm{kg} / \mathrm{d}$ ) or MMF (1-2 g/d in divided doses), and low-dose oral corticosteroids ( $<10 \mathrm{mg} / \mathrm{d}$ prednisone equivalent) ${ }^{14}$. A study has shown that MMF is more effective than azathioprine in maintenance therapy for preventing relapse, with no difference in clinically important side effects $^{21}$. A regular evaluation should be performed to evaluate the patient's response to treatment.

\section{CONCLUSION}

The authors emphasize the importance of this LN class IV case due to the concomitant presence of ANCA-proteinase 3 and the weight of the histopathological pattern when choosing initiation therapy. Both CIPH and MMF, in combination with corticosteroid, are effective options for initial therapy. In this young patient we probably would have chosen MMF as first-line therapy because of the absence of crescents and vasculitis in the renal biopsy. Because of the patient's particular histopathological pattern with the presence of ANCAProteinase 3 , it was decided that a protocol containing cyclophosphamide for initial therapy would be the best option. For the maintenance therapy, the patient initiated MMF with an oral corticosteroid with slow tapering. It is crucial to maintain a regular evaluation to rapidly detect signs of kidney function deterioration that can lead us to a redirection in therapeutic strategy with or without performing a new kidney biopsy to guide further treatment. In this case, after 2.5 years of immunosuppressive therapy, kidney function, proteinuria and lupus activity had an important decrease without significant complications.

\section{Acknowledgements}

The authors would like to thank Dr. Helena Viana of the Nephrology Department of Curry Cabral Hospital for her invaluable contribution.

Disclosure of potential conflicts of interest: none declared.

\section{References}

1. Alarcón GS. Multiethnic lupus cohorts: what have they taught us? Vol. 7, Reumatologia clinica. Spain; 2011. p. 3-6.

2. McClure M, Jones R. Update on lupus nephritis for GPs. Lupus. 2018;27(1):11-14.

3. Hsu C-Y, Chiu W-C, Yang T-S, Chen C-J, Chen Y-C, Lai H-M, et al. Age- and gender-related long-term renal outcome in patients with lupus nephritis. Lupus [Internet]. 2011;20(11):1135-1141.

4. Amann K. Do ANCAs make the difference in lupus nephritis? Kidney Int. 2017;92(5):1048-1050. Available from: https://doi.org/10.1016/j.kint.2017.07.011 
5. Wang Y, Huang X, Cai J, Xie L, Wang W, Tang S, et al. Clinicopathologic characteristics and outcomes of lupus nephritis with antineutrophil cytoplasmic antibody: a retrospective study. Medicine (Baltimore). 2016;95(4): e2580

6. Chen S, Tang Z, Zhang Y, Liu Z, Zhang H, Hu W, et al. Significance of histological crescent formation in patients with diffuse proliferative lupus nephritis. Am J Nephrol. 2014;38(6):445-452.

7. The American College of Rheumatology response criteria for proliferative and membranous renal disease in systemic lupus erythematosus clinical trials. Arthritis Rheum. 2006 Feb:54(2):421-432.

8. Gourley MF, Austin HA 3rd, Scott D, Yarboro CH, Vaughan EM, Muir J, et al. Methylprednisolone and cyclophosphamide, alone or in combination, in patients with lupus nephritis. A randomized, controlled trial. Ann Intern Med. 1996 Oct;125(7):549-557.

9. Donadio JVJ, Holley KE, Ferguson RH, Ilstrup DM. Treatment of diffuse proliferative lupus nephritis with prednisone and combined prednisone and cyclophosphamide. N Engl J Med. 1978 Nov;299(21):1151-1155.

10. Boumpas DT, Austin HA 3rd, Vaughn EM, Klippel JH, Steinberg AD, Yarboro $\mathrm{CH}$, et al. Controlled trial of pulse methylprednisolone versus two regimens of pulse cyclophosphamide in severe lupus nephritis. Lancet (London, England). 1992 Sep;340(8822):741-745.

11. Austin HA 3rd, Klippel JH, Balow JE, le Riche NG, Steinberg AD, Plotz PH, et al. Therapy of lupus nephritis. Controlled trial of prednisone and cytotoxic drugs. N EngI J Med. 1986 Mar;314(10):614619.

12. Houssiau FA, Vasconcelos C, D'Cruz D, Sebastiani GD, De Ramon Garrido E, Danieli MG, et al. Immunosuppressive therapy in lupus nephritis: The Euro-Lupus Nephritis Trial, a randomized trial of low-dose versus high-dose intravenous cyclophosphamide. Arthritis Rheum. 2002; 46(8): 2121-2131

13. Houssiau FA, Vasconcelos C, D'Cruz D, Sebastiani GD, De Ramon Garrido E, Danieli MG, et al. The 10-year follow-up data of the Euro-Lupus Nephritis Trial comparing low-dose and high-dose intravenous cyclophosphamide. Ann Rheum Dis. 2010; 69(1): 61-64

14. Cattran DC, Feehally J, Cook HT, Liu ZH, Fervenza FC, Mezzano SA, et al. Kidney disease: Improving global outcomes (KDIGO) glomerulonephritis work group. KDIGO clinical practice guideline for glomerulonephritis. Kidney Int Suppl. 2012;2(2):139-274.
15. Appel GB, Contreras G, Dooley MA, Ginzler EM, Isenberg D, Jayne D, et al. Mycophenolate mofetil versus cyclophosphamide for induction treatment of lupus nephritis. J Am Soc Nephrol. 2009; 20(5): 1103-1112

16. Bertsias GK, Tektonidou M, Amoura Z, Aringer M, Bajema I, Berden JHM, et al. Joint European League Against Rheumatism and European Renal Association-European Dialysis and Transplant Association (EULAR/ERA-EDTA) recommendations for the management of adult and paediatric lupus nephritis. Ann Rheum Dis. 2012 Nov; 71(11):1771-1782.

17. Hahn BH, McMahon MA, Wilkinson A, Wallace WD, Daikh DI, Fitzgerald JD, et al. American College of Rheumatology guidelines for screening, treatment, and management of lupus nephritis. Arthritis Care Res (Hoboken). 2012 Jun;64(6):797-808.

18. Soares PMF, Borba EF, Bonfa E, Hallak J, Corrêa AL, Silva CAA. Gonad evaluation in male systemic lupus erythematosus. Arthritis Rheum. $2007 \mathrm{Jul} ; 56$ (7):2352-2361.

19. Imran TF, Yick F, Verma S, Estiverne C, Ogbonnaya-Odor C, Thiruvarudsothy S, et al. Lupus nephritis: an update. Clin Exp Nephrol. 2016;20(1):1-13.

20. Walsh M, James M, Jayne D, Tonelli M, Manns BJ, Hemmelgarn BR. Mycophenolate mofetil for induction therapy of lupus nephritis: a systematic review and meta-analysis. Clin J Am Soc Nephrol. 2007 Sep;2(5):968-975

21. Henderson LK, Masson P, Craig JC, Roberts MA, Flanc RS, Strippoli GFM, et al. Induction and maintenance treatment of proliferative lupus nephritis: A meta-analysis of randomized controlled trials. Am J Kidney Dis. 2013; 61(1): 74-87

\section{Correspondence to:}

Marcelo Sousa Aveiro, MD

Nephrology Department, Hospital Central do Funchal, Funchal, Portugal

Avenida Luís de Camões, no 57, 9400-514, Funchal

E-mail: marceloaveiro90@gmail.com 